\title{
La négociation de Maastricht vue de Paris
}

\author{
Georges SAUNIER
}

Le traité de Maastricht, dont les effets, notamment l'instauration d'une monnaie unique, continuent de susciter divers commentaires, est désormais une référence dans l'histoire de l'intégration européenne. En nous intéressant à cette négociation vue du côté français, à la préparation et au déroulement du Conseil européen qui eut lieu dans la petite ville néerlandaise, nous souhaitons contribuer à son étude.

Pour réaliser ce travail, nous nous sommes appuyés sur différentes sources: les archives de la Présidence de la République, ${ }^{1}$ quelques documents provenant d'archives privées, la presse et les média audiovisuels, des témoignages d'acteurs politiques ou administratifs français.

Nous rappellerons d'abord différents éléments de contexte qui constituent, à nos yeux, l'atmosphère historique dans laquelle évoluent les différents acteurs français. $\mathrm{La}$ «fin de l'Europe dite de Yalta» reste à nos yeux un élément déterminant et explicatif de ce contexte. ${ }^{2}$ Que deviendrait l'OTAN dans cet après-guerre froide? Comment organiser la future sécurité en Europe? Comment mettre en œuvre au niveau européen, c'est-à-dire entre les Douze, une défense autonome des autres grands blocs? Telles étaient les questions auxquelles la diplomatie française était confrontée et qui expliquent la volonté de créer une Politique étrangère et de sécurité commune (PESC) traitant aussi des questions de défense.

La fin de la guerre froide impliquait aussi de renforcer l'Europe en tant qu'espace de paix, de coopération économique, culturel et diplomatique. Il s'agissait d'une nécessité, alors que la disparition du glacis soviétique faisait craindre un réveil des nationalismes, comme la tragédie yougoslave naissante pouvait le laisser penser. ${ }^{3}$ Pour les autorités françaises, la réponse résidait dans l'émergence de nouvelles structures paneuropéennes au sein desquelles l'Est et l'Ouest de l'Europe se retrouveraient. Ainsi, en sus des démarches au sein de la CSCE, Paris proposa la création d'une Confédération européenne qui permettrait d'établir un dialogue permanent entre une Communauté européenne renforcée, d'une part, et les pays d'Europe centrale et orientale (PECO), d'autre part. Ces derniers, en effet, ne pouvaient dans l'immédiat adhérer à la Communauté européenne, leurs économies n'y étant pas prêtes. Ainsi, au moment où il fut négocié, le projet d'Union européenne (UE) n'avait donc pas

1. Notamment les fiches résumant la négociation et destinées au Président de la République et plusieurs documents préparant la position française.

2. IFM [Archives Institut François Mitterrand], Fonds Service de presse, Allocution de Monsieur François Mitterrand, Président de la République, lors de la signature de la "Charte de Paris pour une nouvelle Europe" par les chefs d'États des trois Républiques baltes, ..., 06.12.1991.

3. AN [Archives nationales], 5AG4/33-1, J. Blot, Réflexions sur la question allemande, 30.10.1989. 
pour objet d'encadrer plusieurs dizaines de pays supplémentaires mais d'être le noyau dur d'un continent lui-même organisé en confédération. ${ }^{4}$

Aux marges de cette Europe, que faire de la Russie? Au moment où la négociation de Maastricht s'est nouée, il était clair que l'organisation politique et territoriale de l'Union soviétique allait connaître de profonds bouleversements. Or, la Russie restait une puissance potentiellement menaçante. Il fallait donc veiller à ce que l'intégration européenne à venir n'inquiétât pas l'ancien géant. Aussi, d'une façon ou d'une autre, les autorités françaises considéraient comme nécessaire d'associer la Russie à la future confédération. ${ }^{5}$

Quant au cœur de cette Europe à construire, organisée autour d'une Communauté européenne renforcée, il devait permettre à la France de vivre en complète solidarité avec ses principaux voisins et en particulier avec la nouvelle Allemagne unifiée, pays restant au cœur des préoccupations diplomatiques françaises. ${ }^{6} \mathrm{Si}$ François Mitterrand ne voulait pas d'une unification allemande incontrôlée, réalisée en dehors de tout accord international, c'est-à-dire à ses yeux potentiellement dangereuse, il en admettait cependant la légitimité, connaissait son caractère «inéluctable» et a œuvré pour qu'elle intervienne selon certaines conditions. ${ }^{7}$ L'une d'elle était que le processus d'unification allemande ne se fasse pas au détriment de l'approfondissement de l'intégration européenne. Au contraire, unification allemande et approfondissement devaient aller de pair. La volonté de progresser vers une Union économique et monétaire (UEM), puis de renforcer l'Union politique (UP) entre les Douze, s'inscrit très exactement dans cette logique : renforcer les liens entre la future Allemagne et les autres États membres - entre l'Allemagne et la France - afin de créer une solidarité irréversible et contraignante entre eux. ${ }^{8}$

\section{Nécessités «communautaires»}

Pour les autorités françaises, le projet d'Union européenne se justifie donc par la nécessité de répondre à la nouvelle situation née de la disparition du bloc soviétique.

4. Sur ce projet de Confédération européenne, voir : J. MUSITELLI, François Mitterrand, architecte de la Grande Europe: le projet de Confédération européenne (1990-1991), in: Revue internationale et stratégique, 82(2011), pp.18-28; M.-P. REY, Europe is our Common home: a study of Gorbachev's diplomatic concept, in: Cold War History, 2(2004), pp.33-66; R. DUMAS, Un projet mort-né: la Confédération européenne, in: Politique étrangère, 3(2001), pp.687-703.

5. On verra notamment sur ce point deux des documents récemment publiés par le Centre d'analyse et de prévision du Quai d'Orsay: J.-M. GUEHENNO, Après l'URSS, 27.08.1991; P. BUHLER, MAE, CEP, La désagrégation de l'Union soviétique, jusqu'où, 02.09.1991, consulté le 13.05.2012, http:// www.diplomatie.gouv.fr/fr/le-ministere/archives-et-patrimoine/putsch-de-moscou-echantillon-de/.

6. Cf. F. BOZO, Mitterrand, la fin de la guerre froide et l'unification allemande. De Yalta à Maastricht, Odile Jacob, Paris, 2005; M. VAÏSSE, C. WENKEL, La diplomatie française face à l'unification allemande, Tallandier, Paris, 2011.

7. AN, CD.177, Hubert Védrine. Réflexions sur la question allemande, 18.10.1989.

8. Les entretiens menés auprès des principaux acteurs de cette négociation confirment tous ce point. 
Néanmoins, ce projet n'est pas ex nihilo. Il s'inscrit dans une histoire communautaire plus ancienne, ayant ses propres logiques. C'est ainsi que l'UEM ou les nouvelles compétences accordées à l'Union s'inscrivent dans une réflexion politique des autorités françaises à l'égard des grands bouleversements que connaissent l'hexagone et son économie depuis les années 1970 et surtout $1980 .{ }^{9}$ À cet égard, la Communauté européenne apparaît ici sous un double aspect, à la fois accélérateur et régulateur. Accélérateur, puisque l'Acte unique a confirmé et amplifié l'internationalisation - ou plus exactement l'européanisation - de l'économie française. Rappelons que le traité de Maastricht fut négocié parallèlement à l'achèvement du grand marché unique prévu pour 1993; régulateur, car en édifiant un cadre politique, diplomatique, juridique et monétaire à l'échelle du marché économique européen, l'idée française était bien de maîtriser les nouveaux processus économiques à l'œuvre, y compris à l'échelle mondiale. ${ }^{10}$ Là encore, il faut rappeler que la négociation du traité de Maastricht est concomitante avec la négociation de l'Uruguay Round. ${ }^{11}$

Comment exercer cette régulation économique? La délégation française jugeait trois dossiers prioritaires pour y parvenir: mettre en œuvre une UEM, poser les bases d'un espace social européen, permettre une politique industrielle commune. D'autres développements étaient possibles - santé, environnement, recherche, grands réseaux - et même souhaitables, à condition toutefois qu'ils restent secondaires. Enfin, divers volets à négocier devaient permettre à la future union de ne pas être qu'un simple espace économique. ${ }^{12}$ La création d'une citoyenneté européenne, d'une politique culturelle commune, répondaient à cette exigence, $\mathrm{y}$ compris la mise en œuvre d'une Politique étrangère et de sécurité commune (PESC). ${ }^{13}$

Comment et qui contrôlerait cette Union? Les perspectives d'une UEM et d'une PESC d'une part, la transformation de la Communauté en Union d'autre part, firent

9. Cf. O. GALLAND, Y. LEMEL (dir.), La nouvelle société française. Trente années de mutation, Armand Colin, Paris, 1998; H. MENDRAS, L. DUBOYS FRESNAY, Français, comme vous avez changé. Histoire des Français depuis 1945, Tallandier, Paris, 2004; L. DIRN, La société française en tendances, 1975-1995. Deux décennies de changement, PUF, Paris, 1998.

10. Voir G. SAUNIER, Le gouvernement français et les enjeux économiques européens à l'heure de la rigueur 1981-1984, et É. BUSSIERE, Le ministère des Finances et les enjeux économiques européens à l'époque de la cohabitation 1986-1988, in: É. BUSSIERE, M. DUMOULIN, S. SCHIRMANN (dir.), Milieux économiques et intégration européenne au XXe siècle. La relance des années quatre-vingt (1979-1992), CHEFF, Paris,2007, pp.109-145 et pp.147-165.

11. Les négociateurs français veillèrent à isoler les problèmes du GATT de ceux de la négociation du traité de Maastricht afin que la première ne vienne pas gêner la seconde. Voir: AN, PHB.8-4, Bastelica; Lafont. Négociations d'Uruguay. Sommet de Maastricht, 06.12.1991; Lafont. L'état des négociations d'Uruguay, 06.12.1991. Les conclusions du Conseil européen de Maastricht y font pourtant référence, prudemment et en rappelant la nécessaire compatibilité des négociations avec «les objectifs de la Communauté». Les références au texte du Traité d'Union européenne (TUE), incluant protocoles et déclarations, ou aux conclusions du Conseil européen de Maastricht (CCE) proviennent respectivement du Journal officiel de la Communauté et du Bulletin mensuel correspondant.

12. AN, PHB.8-4, Bastelica. Nouvelles compétences, s.d.; voir aussi par exemple IFM, Conférence de presse du Président de la République. Politique étrangère de la France, 18.05.1989.

13. Sur ces points, cf. infra. 
de cette question dite de «l'architecture» un enjeu spécifique de la négociation de Maastricht. À Paris, la réflexion sur ce thème existe dès le printemps $1990 .{ }^{14}$ Ses résultats sont perceptibles dans certains propos de François Mitterrand. ${ }^{15}$ Avant d'en étudier les effets lors de la négociation, il convient d'en rappeler les fondements. Ils tiennent en deux mots: légitimité, finalité.

Mitterrand considérant que la cohérence politique européenne ne pourrait être obtenue qu'avec «beaucoup de patience», il est clair que lors de la négociation de Maastricht, les autorités françaises considéraient toujours l'espace national comme le seul espace politique de référence. ${ }^{16}$ La seule légitimité possible dans le processus de décision communautaire ne pouvait donc être que celle des États. D'où la volonté française, lors de la négociation de Maastricht, de privilégier l'architecture dite du Temple plutôt que celle de l'Arbre. ${ }^{17}$ Le Temple où le Conseil européen, c'est-à-dire la réunion des chefs d'État et de gouvernements, directement responsables devant leurs peuples, reste maître d'œuvre. Quant à la finalité ultime de l'Union, il faut là encore se reporter aux déclarations et actes de Mitterrand. ${ }^{18}$ Celui-ci ne ménage pas son soutien à de grands projets européens tels que l'UEM ou la PESC. Il n'hésite pas à parler de citoyenneté européenne et se déclare favorable à «un système à finalité fédérale». Pour autant, il ne faut pas se tromper sur le sens de ces termes. À ses yeux, l'intégration européenne ne peut qu'être respectueuse des intérêts et différences des États membres, de leurs institutions. Il se refuse à les voir se fondre «dans la grisaille». En d'autres termes, la dualité du système de décision communautaire, à la fois supranational et intergouvernemental, reste à ses yeux la bonne formule et explique son soutien à des propositions permettant de mieux articuler les niveaux nationaux et communautaires, tels que la subsidiarité, le congrès des Parlements, ou les arbitrages quant aux procédures de votes à l'unanimité ou à la majorité.

Quoi qu'il en soit de ces considérations, les autorités françaises se concentrèrent prioritairement lors des négociations du traité de Maastricht sur deux grands dossiers: la PESC et l'UEM. Le projet de PESC, nous l'avons indiqué, répond au nouveau contexte international et apparait comme le prolongement des premiers efforts de coordination des diplomaties des États membres, nés dans les années soixante-dix, codifiés et intensifiés depuis. Avec la PESC, il s'agissait dans l'esprit des autorités

14. Voir Archives privées, Elisabeth Guigou (EG). Quel pourrait être le contenu d'une initiative sur l'Union politique de l'Europe, 20.03.1990.

15. Le débat sur les institutions européennes en France est toutefois plus large et plus complexe. Voir B. DENNI, Du référendum du 20 septembre 1992 sur l'Union européenne aux élections législatives de mars 1993, in: P. HABERT, P. PERRINEAU, C. YSMAL (dir.), Le vote sanction. Les élections législatives des 21 et 28 mars 1993, Presses de la Fondation nationale des sciences politiques, Paris, 1993, pp.91-109.

16. IFM, Conférence de presse de Monsieur le Président de la République à l'issue du Conseil européen, Dublin, 26.06.1990.

17. AN, PHB.8-4, Caroline de Margerie (CDM). La structure du traité, 06.12.1991.

18. Les déclarations de ce dernier à ce sujet sont rares. 
françaises de franchir une étape supplémentaire et de jeter les bases d'une diplomatie et d'une défense européenne autonome, notamment à l'égard des États-Unis. ${ }^{19}$

L'autre grand projet - sans doute le principal - était celui d'UEM. ${ }^{20}$ La France est de longue date attachée à ce projet, comme l'atteste le rôle joué par Paris lors de la mise en place du Système monétaire européen (SME). Lorsque les Socialistes français arrivèrent au gouvernement en 1981, ils cherchèrent d'ailleurs à en renforcer les mécanismes. ${ }^{21}$ Les dévaluations de 1981, 1982 et 1983, les exigences de la politique du franc fort, les mouvements financiers erratiques américains, leur permirent de mesurer la faiblesse du franc, l'influence monétaire de l'Allemagne et la puissance du dollar. ${ }^{22}$ Autant d'éléments qui militaient en faveur de l'UEM, grâce à laquelle on espérait desserrer l'étau du «mark» et lutter contre la suprématie du dollar, sans oublier les gains possibles pour les entreprises françaises bénéficiant d'une monnaie unique dans un marché unique. Les concessions faites aux autorités allemandes en 1989/1990 - sur la libéralisation des capitaux ou l'indépendance de la Banque centrale européenne $(\mathrm{BCE})$ - s'expliquent par cet impératif: constituer un système de solidarité monétaire européen et y faire entrer le deutschemark. ${ }^{23}$

\section{Équilibres et déséquilibres intérieurs}

Les décideurs politiques français ont dû tenir compte du jeu politique national qui leur donnait plus ou moins de latitude lors des négociations. Sur cette période 1988-1991, les Socialistes ne sont pas majoritaires à eux seuls à l'Assemblée et doivent tour à tour composer avec le Parti communiste, les députés non-inscrits ou centristes. ${ }^{24}$ Deux gouvernements se succèderont: ceux de Michel Rocard (1988-1991) et d'Édith Cresson (1991-1992). Au moment du référendum, Pierre Bérégovoy - ministre de l'Économie et des Finances depuis 1984, artisan du franc fort et négociateur de l'UEM - deviendra Premier ministre.

19. Cf. E. REMACLE, Les dispositions du Traité de Maastricht: politique étrangère et sécurité commune de l'Union européenne, in: Études internationales, 2(1992), pp.377-394; D. DESCHAUXBEAUME, De l'Eurocorps à une armée européenne? Pour une sociologie historique de la politique européenne de sécurité et de défense (1991-2007), IEP Grenoble, Doctorat [inédit], 2008.

20. Voir K. DYSON, K. FEATHERSTONE, The Road to Maastricht. Negotiating Economic And Monetary Union, Oxford University Press, Oxford, 2000; M.-T. BITSCH, Histoire de la construction européenne de 1945 à nos jours, Éd. Complexe, Bruxelles, 2004.

21. AN, 2233, Delors. Le Conseil européen du 26.11 et le volet relance, 10.11.1981; Ministère de l'Économie et des Finances, compte-rendu des conversations franco-allemandes, 04.11.1981.

22. Interviews de l'auteur avec Elisabeth Guigou, Hubert Védrine, Jean-Louis Bianco, Philippe Jurgensen.

23. Sur la négociation UEM, voir AN 5AG4-EG.22-4, 22-10, 88, 89, 90-1, 91-1.

24. Voir P. FAVIER, M. MARTIN-ROLAND, La Décennie Mitterrand, t.3 et 4, Seuil, Paris, 1998-1999; J.-J. BECKER, P. ORY, Nouvelle histoire de la France contemporaine, t.19, Seuil, Paris, 2002. 
Commencées dans la confiance, les négociations de Maastricht se terminent dans l'hostilité de l'opinion publique. La cote de popularité du Président, entre 50 et $60 \%$ d'opinions favorables en mai 1991 , tombe à 30-40\% à l'été $1992 .{ }^{25}$ Or, l'engagement européen de Mitterrand, le cœur de sa campagne présidentielle en 1988 où il n'hésitait pas à déclarer que «le principal est que l'Europe avance», constituait l'un des ressorts de son action politique. ${ }^{26}$ Mais c'est un ressort de plus en plus contesté à l'extrême gauche - Parti communiste - comme à l'extrême droite - le Front national -, le tout dans un contexte politique de plus en plus difficile, ce que révélera le difficile débat au moment du référendum sur le traité. Cette évolution eut pour conséquences de réduire les marges de manœuvre du gouvernement lors des négociations mais aussi de rendre nécessaire un succès sur la scène diplomatique à présenter à l'opinion publique.

Nous avons présenté les éléments de contexte précédents de façon séparée. Il est vrai que les comptes rendus de discussion, les notes des conseillers, les discours publics, les font apparaitre séparément. Néanmoins, ces sujets sont traités, in fine, par les mêmes autorités politiques, dans les mêmes temps et lieux. Bref, on gardera à l'esprit leur globalité.

Négocié dans une France en mutation, par un gouvernement chahuté sur le plan intérieur, dans un contexte international mouvant et fondamentalement marqué par la fin de la Guerre froide, les autorités françaises avaient pour objectif la construction d'une Union européenne à Douze, très intégrée autour d'une monnaie, d'une diplomatie et d'une défense commune, entourée par une Confédération européenne plus vaste et respectueuse de la diversité des États.

\section{9-1991 - Négocier le Traité d’Union Européenne}

Qui furent les négociateurs de ce traité? Une trentaine de personnes si l'on compte les hauts fonctionnaires capables d'avoir une vue d'ensemble, quelques ministres dont les fonctions ou le poids politique les plaçaient en situation de prise de décision sur les textes négociés, les arbitrages étant rendus in fine par le Président de la République. ${ }^{27}$

Les profils sont divers: Président, Premier ministre, ministres, conseillers, hautsfonctionnaires des ministères ou diplomates siégeant dans les instances communautaires. Lors du Conseil européen de Maastricht, en décembre 1991, Matignon est aux mains d'une femme, Édith Cresson, ancien ministre délégué aux Affaires européennes. Roland Dumas, ministre des Affaires étrangères et Pierre Bérégovoy, ministre de l'Économie et des Finances, sont directement associés et proches du Président. Le ministre délégué aux Affaires européennes, Élisabeth Guigou, joue un grand rôle.

25. IFM, Dossier Sondages.

26. IFM, Mitterrand. Lettre à tous les Français, avril 1988.

27. La liste a été établie à partir de différents comptes rendus de réunions dont $\mathrm{AN} / 54,61,62$. 
Elle était précédemment à l'Élysée, conseillère pour les questions européennes. D'autres ministres sont bien entendu associés du fait de leurs portefeuilles et de leurs poids politiques. Quant aux conseillers et hauts-fonctionnaires, ils proviennent des principaux ministères et de la Présidence de la République. ${ }^{28}$ Ces hommes - principalement - et femmes maitrisaient alors parfaitement les rouages communautaires. Ils en avaient une solide expérience, des habitudes de travail et connaissaient leurs interlocuteurs.

Quand négocièrent-ils? La question suppose de déterminer une date de départ, ce qui est délicat puisque, malgré ses aspects novateurs, le traité de Maastricht s'inscrit dans une continuité. D'une certaine façon, l'Acte unique a ouvert à la discussion nombre des débats qui se sont concrétisés à Maastricht. Il est certain, toutefois, qu'au tournant des années 1988/89, Paris suit de près les efforts de Jacques Delors en faveur de l'UEM et agit en conséquence. Lors du Conseil européen de Strasbourg, en décembre 1989, Mitterrand obtient du Chancelier Helmut Kohl qu'une date soit fixée pour que débute la CIG sur l'UEM. ${ }^{29}$ La révolution de velours et le processus d'unification allemande agissent dès lors comme un accélérateur. Le projet d'UP devient progressivement une évidence: de chaque côté du Rhin, on s'accorde pour qu'intervienne une nouvelle phase de l'intégration communautaire. En avril 1990, la première lettre Mitterrand-Kohl est envoyée.

Quelle fut la tactique française dans cette négociation? ${ }^{30}$ D'abord, «surveiller de près» l'Allemagne et à chaque étape de la négociation s'assurer de disposer d'un accord solide avec ce pays. ${ }^{31}$ L'affaire pouvait être délicate dans la mesure où la RFA pouvait prendre des postures contradictoires. ${ }^{32}$ D'où, tout au long de la négociation, un dialogue constant entre le Président et le Chancelier, leurs collaborateurs et les deux ministres des Affaires étrangères. À partir de là, construire un rapport de force pour faire face à la Grande Bretagne. Ce pays, en effet, ne voulait pas de cette négociation, n'avait donc aucune marge de manœuvre, ferait tout pour diviser l'Allemagne et la France et tenterait de faire reporter les principales décisions. Il fallait donc l'isoler, ne rien lui concéder d'essentiel, mais sans pour autant l'amener à la rupture, ... tout en menaçant de le faire. Telle était la quadrature du cercle.

28. Citons notamment Pierre de Boissieu (Affaires étrangères, représentant français à la CIG sur l'UP), Jean-Claude Trichet (Économie, CIG sur l'UEM), Caroline de Margerie, Philippe Bastelica, Pierre Morel, Hubert Védrine (Présidence de la République), François Lamoureux (Matignon), Bernard Kessedjan, Jean-Michel Casa, Alain Dejammet (Affaires étrangères), Pierre Vimont (Affaires européennes), François Villeroy de Galhau (Économie), Jean Vidal (COREPER), Bernard Vial (SGCI) et Renaud Denoix de Saint Marc (SGG).

29. Les deux CIG, sur l'UEM et l'UP, débutèrent en décembre 1990.

30. Voir notamment: AN, CDM.14-1, Boissieu. Position des délégations, 25.11.1991; PHB.9-1, Boissieu, Conseil européen: union politique, 22.11.1991; PHB.9-1, Casa. Conférence intergouvernementales: Conseil restreint du mercredi 27 novembre, 26.11.1991; CDM.14-1, CDM, Note manuscrite, 12h, s.d.; CDM.14-1, CDM, Entretien avec M. Lubbers, 27.11.1991; CDM.14-1, EG. Note manuscrite, s.d.

31. AN, CDM.14-1, P. Boissieu, Position des délégations, 25.11.1991.

32. La délégation allemande se déclarait facilement favorable au communautaire mais réclamait l'unanimité sur l'industrie, le social voir même la culture. 
Comment y parvenir? En établissant un accord à onze délégations. Un tel accord rendraient le coût d'une rupture - et donc d'un possible isolement - très coûteux pour la Grande Bretagne. Sur quelle délégation pouvait compter la France pour y parvenir en dehors de la RFA? À la veille du sommet, Pierre de Boissieu dresse le portrait des différents partenaires tel qu'il a pu le constater pendant la conférence intergouvernementale. Portrait largement partagé et qui témoigne de l'atmosphère au sein de la délégation française ainsi que des rapprochements envisageables lors des discussions. L'Espagne? «Un grand. Mais intraitable», souvent proche des positions françaises, mais dont certaines demandes ne pouvaient être satisfaites. ${ }^{33}$ Le Danemark? Ils sont les véritables «Français de Scandinavie», excellent soutien. Le Luxembourg? «Remarquable». La Belgique? Proche de Paris, le pays traversait toutefois des difficultés gouvernementales paralysantes. Heureusement, son négociateur était «de talent». L'Irlande ne posait pas de problème particulier, il fallait lui trouver une formulation correcte sur la défense. La présidence néerlandaise? Elle s'était d'abord opposée à Paris et Bonn mais, depuis octobre 1991 «jou[ait] le jeu». Plus délicat semblaient le cas de l'Italie, «absente»; du Portugal, dans une opposition résolue à Paris; et de la Grèce, uniquement intéressée par les transferts financiers et par son adhésion à l'Union de l'Europe occidentale (UEO). Maintenir un front uni serait donc délicat. Il faudrait circonscrire les «ayatollahs», "gardiens de la vraie foi» communautaire, qui en demanderaient toujours plus au risque de faire échouer l'accord; éviter les «zélés» maintenant jusqu'au bout leurs demandes excessives; se méfier des «temporisateurs», toujours prompts à renvoyer les difficultés à plus tard et finalement à vider les textes de leur substance. À l'inverse de ceux là, il fallait aller vite et prendre les décisions nécessaires.

À cet égard, la France pouvait être victorieuse, mais elle avait un «talon d'Achille». Étant la seule à pourvoir «signer le texte tel qu'il est présenté par la présidence [néerlandaise]» à la veille du Conseil européen de Maastricht, elle devait veiller à maintenir l'équilibre général du texte qui lui paraissait favorable. Le risque était que les Britanniques et les Allemands ne se livrent à une guerre de tranchée sur l'architecture de l'Union, les pouvoirs du Parlement ou les affaires de justice. Que les Allemands lâchent les Français sur les questions de défense. Que les Espagnols réclament trop de compensations budgétaires.

Restait le problème de la Commission. Le constat de Paris était sévère: «peut être la seule négociation communautaire que la Commission n'ait pas prévue, pas comprise, pas maitrisée». Surtout, elle s'opposait par la voix de son Président, Jacques Delors, à la structure en Temple et réclamait celle de l'Arbre où la Commission gardait le contrôle, y compris dans les domaines aussi sensibles que la monnaie, la diplomatie ou la défense. Afin de neutraliser l'opposition de la Commission, Mitterrand rencontra à plusieurs reprises Delors. Mais jusqu'à la veille du Conseil européen de Maastricht, la France ne put compter sur le soutien de Bruxelles. ${ }^{34}$

33. Pour les citations de ce paragraphe, voir AN, CDM.14-1, Boissieu. Position des délégations, 25.11.1991.

34. AN, PHB.9-1, CDM. Entretien avec M. Jacques Delors, 05.12.1991. 
Aller vite, ne pas s'enliser dans de trop longues négociations, tel semblait être le leitmotiv français. Aller vite, c'est-à-dire faire ratifier le traité dans le courant de l'année 1992, avant que ne commencent d'autres délicates négociations communautaires sur l'agriculture et le budget - le futur «paquet Delors II», qui mettraient le couple franco-allemand en difficulté. ${ }^{35}$ On peut distinguer trois grandes étapes.

Premièrement, du mois d'avril à la fin de l'année 1990, la priorité française fut de faire adopter un mandat de négociation pour les deux CIG, proche des propositions franco-allemandes. Ce qui conduisit à de nombreux contacts franco-allemands. Lors du Conseil européen de Rome, en décembre, la délégation française eut le sentiment d'avoir atteint ce premier objectif. La deuxième phase de la négociation débuta avec la présidence luxembourgeoise, durant le premier semestre 1991. La disparition de l'Union soviétique, la crise du Golfe et surtout les débuts de celle de Yougoslavie ${ }^{36}$ compliquèrent l'agenda de la négociation mais la période permit à la délégation française d'affiner ses positions et de les défendre au niveau communautaire. ${ }^{37}$ Toutefois, à mesure qu'approchait l'échéance du Conseil européen de Luxembourg, les 28-29 juin 1991, il devint clair que l'on ne pourrait pas conclure. Paris réussit cependant à faire acter, avec l'aide de l'Allemagne, un certain nombre de points. $\mathrm{La}$ troisième phase des négociations s'ouvrait donc avec le second semestre 1991.

\section{Le Conseil européen de Maastricht}

Prévu pour les 9-10 décembre 1991, c'est donc à l'occasion du Conseil européen de Maastricht, au terme de la présidence néerlandaise du second semestre 1991, que les Douze devraient amender puis adopter les conclusions des deux CIG. Ce texte serait la base du futur traité. Ruud Lubbers, le Premier ministre néerlandais, président en exercice de la Communauté, soulignait par avance l'«importance historique» de la rencontre et les «grands espoirs» qu'elle soulevait. ${ }^{38}$ Comment la délégation française se présentait-elle à cette échéance?

Il avait d'abord fallu se battre avec la présidence néerlandaise. Dans le courant du mois d'août, en effet, celle-ci avait mis deux textes en circulation. Ces deux textes balayaient d'un trait toutes les positions françaises pourtant actées à Luxembourg et remplaçaient en particulier le Temple par l'Arbre. Face à ce que certains à Paris

35. AN, PHB.8-4, PHB. La cohésion, 06.12.1991.

36. Les divergences entre Paris et Bonn sur ce dossier sont connues. Il fut cependant décidé qu'elles ne troubleraient pas la négociation de Maastricht. Un texte fut néanmoins adopté en conclusion du Conseil. AN, PHB.8-4, Morel. Situation en Yougoslavie: aide humanitaire et gestes politiques, 07.12.1991; Conseil européen de Maastricht (09.-10.12.1991) - Yougoslavie, 06.12.1991.

37. Ce fut le cas pour la PESC, l'architecture institutionnelle de l'Union, le contenu concret de l'UEM (monnaie unique et non commune, structures de décision, durée et dates des différentes phases de transition), ainsi que sur différents dossiers tels que la culture, l'industrie et le social.

38. AN, PHB.8-4, TD La Haye 1003, 05.12.1991. 
considéraient comme une déclaration de guerre diplomatique, ${ }^{39}$ l'Élysée et la Chancellerie imposèrent que l'on reprenne les négociations à partir des conclusions de juin, les documents néerlandais étant rejetés lors d'un Conseil des ministres, «le 30 septembre [1991], par 10 voix contre 2 », «sous les sifflets». ${ }^{40}$ Puis, à la mi-octobre, une nouvelle lettre Mitterrand-Kohl fut adressée aux Dix précisant ce que devait être la future PESC. Mais plusieurs semaines avaient été perdues. Le mois de novembre devrait donc être décisif. Paris chercha dès lors à faire le tri dans les demandes des autres délégations sans renoncer à ses propres priorités: annuler les chapitres sur l'énergie (trop libéral), la protection civile et le tourisme; conserver ceux sur la santé, la protection des consommateurs, la recherche, les réseaux transeuropéens et l'environnement. ${ }^{41}$ Enfin, trancher ce qui devait être ou non décidé à la majorité.

À cet effet, les contacts se multiplièrent à tous les niveaux. ${ }^{42}$ L'idée étant que la présidence communique à l'occasion du Conseil européen de Maastricht un texte largement agréé, ne comportant plus que quelques points à régler, afin d'éviter un nouveau report des discussions. Mitterrand rencontra plusieurs de ses homologues. Des conclaves ministériels eurent lieu au niveau communautaire de la mi-novembre au début décembre. À Paris, plusieurs réunions de haut-niveau arrêtèrent les positions du gouvernement. ${ }^{43}$ Le Conseil européen pouvait dès lors se tenir avec quelques chances de conclure. Pour Mitterrand, ce Conseil débuta le dimanche 8 au soir par un dîner avec le Président du Conseil italien. Il prit fin vers 2 heures du matin, le mercredi 11 décembre après un marathon exténuant. Quels en étaient les enjeux pour la France?

Conformément à l'ordre du jour prévu, la première séance plénière, le 9 décembre 1991 dans la matinée, conduisit les Douze à traiter la question de l'Union économique et monétaire.$^{44}$ Dans ce dossier, la France estimait avoir concédé l'essentiel, notamment sur l'indépendance de la Banque centrale européenne (BCE). Un accord permettant la création d'une monnaie unique semblait donc à portée de main. Encore

39. Entretien avec Roland Dumas, 04.08.2000.

40. AN, CDM.14-1, Boissieu. Position des délégations, 25.11.1991; AN, CDM.14-1, CDM. Entretien avec M. Lubbers 27.11.1991.

41. AN, PHB.8-4, Nouvelles compétences, s.d.; PHB.9-1, Casa. Conférence intergouvernementale: Conseil restreint du mercredi 27.11, 26.11.1991.

42. Par exemple AN, CDM.14-1, Entretien avec M. Lubbers, op.cit.; CDM. Dîner avec M. Andreotti à Maastricht, 05.12.1991; PHB.9-1, Entretien avec Delors, op.cit.; CDM.14-1, Morel. Votre déjeuner avec M. Major le 2.12 à Londres, 25.11.1991.

43. AN, PHB.9-1, Casa. Conférence intergouvernementales: Conseil restreint du mercredi 27 novembre, 26.11.1991; CDM.14-1, Conseil restreint. Préparation du Sommet de Maastricht, 27.11.1991; CDM.14-1, Lamoureux. L'union politique. Dossier pour la réunion ministérielle de préparation du Sommet de Maastricht ..., 26.11.1991; CDM.14-1, Secrétariat général du gouvernement. Compte rendu de la réunion de ministres tenue le 28.11.1991 à l'hôtel Matignon, 02.12.1991; CDM.14-1, Compte rendu de la réunion interministérielle le lundi 25.11.1991, 26.11.1991.

44. AN, PHB.8-4, CDM. Conseil européen de Maastricht, 07.12.1991; TD La Haye 1003, 05.12.1991. Il existe deux documents antici, mais incomplets, des discussions du Conseil européen de Maastricht dans les archives de la Présidence françaises. Nous nous y référons avec la mention antici, AN, CDM.14-1, Notes manuscrites; Présidence de la République, Conseil européen (Maastricht, 09.-10.12. 1991). 
fallait-il que les textes définissant les modalités de passage à la troisième phase de l'UEM - c'est-à-dire l'étape où les pays respectant les critères de convergence économique abandonnaient leurs monnaies nationales pour adopter la monnaie unique soient suffisamment contraignants pour que les décisions prises ne restent pas «lettre morte». ${ }^{45}$ Or, malgré d'ultimes tractations menées par Bérégovoy, le mécanisme aboutissant au lancement de cette $3 \mathrm{e}$ phase restait complexe: plusieurs modes de décision, à l'unanimité ou à la majorité, à des dates plus ou moins éloignées, avec plus ou moins de pays. La procédure restait donc aléatoire. Pour la délégation française et Mitterrand lui-même, la priorité était que le traité rende la création de la monnaie unique «irréversible». ${ }^{46}$

C'est dans ces conditions qu'eut lieu, la veille du Conseil européen, un discret dîner entre François Mitterrand et Gulio Andreotti. ${ }^{47}$ Rapidement, les deux hommes se sont entendus sur une idée simple: en séance plénière, le lendemain, ils proposeraient de fixer, dans le traité, une date butoir à partir de laquelle, quoi qu'il arrive, les États membres respectant les critères de convergence passeraient à la troisième phase, et ceci indépendamment de toutes autres considérations ou votes. ${ }^{48}$ L'idée, qui circulait depuis quelques temps et que les Français avaient travaillée, avait ses chances d'être retenue. ${ }^{49}$ Le lendemain, en séance plénière, après d'ultimes discussions sur cette question, Mitterrand, soutenu par Andreotti, proposa comme convenu de «fixer une date de manière précise », début $1999 .{ }^{50}$ La proposition franco-italienne emporta l'adhésion des autres délégations, notamment de l'Allemagne prévenue à l'avance. De fait, le passage à la troisième phase, prévu pour le 1er janvier 1999, devenait «irréversible», aucun État membre ne pouvant désormais en empêcher la réalisation. Il s'agissait d'une importante victoire pour la délégation française, Mitterrand s'en félicitant dans sa conférence de presse au terme du Conseil. ${ }^{51}$

Restait cependant à surmonter l'opposition britannique. Dès le début de la CIG sur l'UEM, Londres avait proposé des solutions alternatives à la monnaie unique, comme par exemple la création d'un SME renforcé. Mais ces tentatives furent un échec et, à Maastricht, John Major avait devant lui un front de onze États décidés.

45. AN, PHB.8-4, PHB. Union économique et monétaire (passage en troisième phase), s.d; PHB. Union économique et monétaire (passage en troisième phase), s.d.; CDM.14-1, EG, Maastricht. Passage à la phase finale de l'Union économique et monétaire, 18.11.1991.

46. AN, CDM.14-1, Secrétariat général du gouvernement. Compte rendu de la réunion de ministres tenue le 28.11.1991, 02.12.1991; CDM.14-1, Compte rendu de la réunion interministérielle le 25.11.1991, 26.11.1991.

47. AN, CDM.14-1, CDM. Dîner avec M. Andreotti à Maastricht (08.12.1991), 05.12.1991.

48. AN, CDM.14-1, CDM. Sujets de Maastricht. Abordés entre le Président de la République et M. Andreotti. Résumé des discussions, 05.12.1991.

49. AN, PHB.8-4, PHB. Union économique et monétaire (passage en 3e phase), s.d.; CDM.14-1, EG, Maastricht. Passage à la phase finale de l'Union économique et monétaire, 18.11.1991; CDM. Points importants des conférences de Maastricht, 29.11.1991; CDM. Conseil restreint du mercredi 27 novembre sur les conférences intergouvernementales, 26.11.1991.

50. AN, CDM.14-1, CDM. UEM - dernier état des négociations, 09.12.1991.

51. IFM, Conférence de presse de M. le Président de la République à l'issue du Conseil européen, 10.12.1991. 
Or, comme le fit remarquer Major, son parlement ne pourrait jamais accepter de ratifier un traité l'obligeant à abandonner la livre. Pour contourner ce problème, Paris avait discuté la possibilité d'une clause d'exemption. La Grande-Bretagne signerait le traité mais déclarerait dans un protocole annexe ne pas s'engager à passer à la troisième phase. La délégation française en acceptait l'idée, à condition que cette clause ne soit pas extensible à d'autres - ce que demandait la Grande-Bretagne -, ce qui aurait remis en cause le caractère irréversible du traité. Bref, la clause devait être très encadrée. Paris comptait sur la RFA et la Commission pour l'appuyer en ce sens. $\mathrm{Au}$ final, après d'âpres discussions dans la matinée du 9 décembre, la Grande-Bretagne dut se résigner à une clause spécifiquement britannique même si, pour des raisons constitutionnelles, le Danemark bénéficia d'un protocole annexe identique.

Concernant l'UEM, quelques autres détails furent discutés en marge du Conseil, notamment la question du siège de la future BCE. Pour Paris, l'affaire était toutefois plus large: traditionnellement, la France refusait en effet de se prononcer sur ces sujets tant que le siège du Parlement européen n'aurait pas été définitivement fixé à Strasbourg. Un accord faillit cependant être trouvé sur ces problèmes de sièges, grâce notamment à l'action de Ruud Lubbers, mais fut finalement repoussé à la fin de l'année 1992, les Douze espérant de nouvelles propositions de Jacques Delors. Ceci, selon un souhait français..$^{52}$

Le résultat obtenu à Maastricht à l'égard de l'UEM mérite d'être souligné. Le gouvernement français et le Président de la République le présentèrent comme une avancée historique conforme aux intérêts français. De fait, le traité fut appliqué et la monnaie unique entra en vigueur à la date prévue. Pour Paris, il s'agissait d'une avancée qualifiée «d'historique», les autorités françaises ayant régulièrement réclamé le renforcement de la solidarité monétaire communautaire. ${ }^{53}$

Le second dossier d'importance discuté à Maastricht est celui de la PESC. Il s'agissait pour Paris d'une «question fondamentale», priorité a priori partagée par l'Allemagne, cœur de la future union politique. ${ }^{54}$ La PESC devait permettre de dépasser la coopération politique (CPE) codifiée par l'Acte unique et faire franchir une nouvelle étape à la diplomatie commune. À l'égard de ce second pilier de la future Union, placé sous le seul contrôle des États, le projet de traité tel que présenté par la Présidence paraissait assez ambitieux aux yeux des Français, y compris dans le domaine si sensible de la défense. ${ }^{55}$ Il était proposé une articulation nouvelle entre la

52. AN, PHB.8-4, CDM. La clause d'exemption ("Opting out"), s.d.; Siège de la banque centrale et de l'Institut monétaire européen, s.d.; CDM. Siège des institutions, 06.12.1991; PHB.9-1, CDM et PHB, Conseil européen de Maastricht. Demandes allemandes, 21.11.1991.

53. Le gouvernement s'emploie à présenter l'accord de Maastricht et en particulier celui sur l'UEM comme «l'acte le plus important depuis le traité de Rome de 1957». AN, PHB.9-1, Lettre de Matignon, Centre d'information et de documentation, décembre 1991.

54. AN, PHB.8-4, CDM. La politique étrangère et de sécurité (pp.104 sqq. du projet de traité), 07.12.1991; CDM. La sécurité et la défense, 07.12.1991.

55. AN, CDM.14-1, Union politique SN 252/91, 09.12.1991. 
future Union, l'UEO et l'OTAN..$^{56}$ Le texte présentait la perspective d'une défense commune. Enfin, il prévoyait que certaines décisions se prendraient à la majorité et définissait une unité d'action «sans réserve» de l'ensemble des États membres. ${ }^{57}$

La France et l'Allemagne - soutenues par l'Espagne, le Luxembourg, la Belgique et la Grèce - avaient fait connaître leurs positions en octobre. Le Conseil européen et le Conseil définiraient à l'unanimité les grandes lignes, les positions et les actions communes de la PESC, mais leurs modalités d'application seraient décidées à la majorité. Ces actions devaient être compatibles avec les engagements spécifiques de chaque État membre: neutralité ou appartenance à l'Alliance atlantique par exemple. C'est cette perspective d'un vote à la majorité qui nourrissait l'opposition de la Grande-Bretagne à la PESC. La délégation britannique maintenait son désaccord sur trois autres points: l'UEO devait rester sous l'autorité de l'OTAN; Londres s'opposait à définir dans une liste ce qui relèverait du domaine de la PESC; il ne devait pas y avoir de référence à une «défense commune». L'opposition était donc frontale, comme les discussions l'attestent. La délégation française - espérant le soutien sans faille de l'Allemagne - étant prête à certaines concessions à condition de ne pas dénaturer le texte : une nouvelle étape devait être franchie dans ce domaine.

$\mathrm{Au}$ final, un compromis fut trouvé, après que Mitterrand et Kohl se soient personnellement prononcés et consultés, le Chancelier précisant lors d'une ultime rencontre entre les deux qu'il fallait «se battre sur la défense». ${ }^{58} \mathrm{La}$ procédure de décision mélangerait effectivement unanimité et majorité, mais la majorité ne s'appliquant qu'à des domaines décidés ... à l'unanimité. Le texte précise cependant qu'un pays ne devait pas, par ce biais, empêcher le bon fonctionnement de la PESC. Compromis, aussi, sur la défense, le texte final enregistrant la formulation proposée par la présidence néerlandaise: la PESC traiterait des questions de sécurité, «y compris la définition à terme d'une politique de défense commune, qui pourrait conduire, le moment venu, à une défense commune». L'expression «politique de défense commune» agréait plusieurs délégations, car elle faisait référence à des discussions - et non actions! - dans ce domaine, voire à celles menées dans le cadre de l'OTAN. Toutefois, le texte fait bien référence à une «défense commune», c'est-à-dire aux modalités concrètes - et non discussions - d'une défense propre à l'Union. Précisément, le traité ajoutait une déclaration des États membres de l'Union de l'Europe occidentale (UEO), celle-ci devenant partie intégrante de l'Union, ce qui convenait parfaitement aux Français qui voulaient faire de cet organisme le bras armé de l'Europe.

Certes, le texte final était un compromis, mais une clause de revoyure existait pour 1996 et les nouveaux développements de la PESC trouvaient une base légale. Une défense existait, certes compatible avec l'Alliance atlantique, mais devant en consti-

56. Le Conseil européen de Maastricht intervient à la suite du sommet de l'OTAN des 7-8 novembre 1991 où fut acté le terme «identité européenne» de défense, «compatible et complémentaire» de l'Alliance atlantique.

57. TUE.

58. AN, PHB.9-1, CDM. Entretien du Président de la République avec le Chancelier Kohl (Maastricht, mardi 10.12.1991, 16h00), s.d. 
tuer à terme «le pilier européen». ${ }^{59}$ La France avait donc atteint ses objectifs, sauf peut-être sur le vote à la majorité. On peut toutefois s'interroger sur la volonté française en ce domaine. Cherchait-on véritablement, à Paris, à créer une diplomatie unique? En guise de réponse, on rappellera que, tout au long de la négociation, la France veilla n'être liée par aucun «mandat impératif» au sein du Conseil de sécurité de l'ONU.60

Quoi qu'il en soit, lors de sa conférence de presse, Mitterrand pouvait déclarer:

«Ce qui a été fait représente un grand pas en avant. Je l'aurais voulu plus grand encore, c'est à dire laisser un peu de côté l'unanimité, retrouver plus souvent les majorités qualifiées et même parfois les majorités simples. C'est comme cela que la Communauté trouvera sa pleine liberté d'action, mais enfin, je dois reconnaitre que l'effort accompli est un effort sérieux et profitable». ${ }^{61}$

Dernier et nouveau pilier, celui dit «Justice et affaires intérieures» (JAI). La France en acceptait le principe. Ce dossier n'était pourtant pas son initiative, mais d'origine allemande. Bonn souhaitait que les États membres accentuent leurs politiques communes à l'égard du contrôle des mouvements migratoires et de la lutte contre le banditisme. Une fois de plus, la Grande Bretagne était réticente, comme elle l'avait été lors de la création de l'espace Schengen. Bonn et Londres s'opposaient notamment sur les modalités de décisions. Les Allemands voulaient communautariser les procédures au maximum; à l'inverse, les Britanniques voulaient inscrire la totalité de ces sujets dans un troisième pilier, strictement intergouvernemental. Le bras de fer, prévisible, inquiétait modérément Paris, la délégation française étant «extrêmement [à] l'aise» sur ce dossier, «n'ayant pas de vraie difficulté» sauf sur les règles du droit d'asile qui nécessitaient une modification du préambule de la Constitution de 1958. ${ }^{62}$ Bref, il s'agissait d'une bonne occasion de soutenir la RFA. Au final, les textes adoptés entérinent de réelles avancées mais restent loin de la communautarisation totale espérée par la RFA.

L'architecture de l'Union fut l'objet de nombreux échanges en séance plénière lors du Conseil. Le dossier était jugé délicat pour Paris qui craignait de voir réapparaitre à cette occasion une irréductible opposition entre Bonn et Londres; opposition qui pourrait empêcher l'accord final. La position française était connue: le Conseil européen devait être l'instance supérieure de l'Union car seuls les États disposaient de la légitimité démocratique nécessaire. Certes la France avait accepté l'indépendance de la $\mathrm{BCE}$, mais Paris avait veillé à ce que ce soit le Conseil qui définisse les «grandes orientations de la politique économique et la nomination des principaux dirigeants de [...] la $\mathrm{BCE} \gg .{ }^{63}$ En d'autres termes, pour la délégation française, il

59. TUE.

60. AN, CDM.14-1, CDM. L'union politique et le Conseil de sécurité, 25.11.1991; PHB.8-4, CDM. La politique étrangère et de sécurité, 07.12.1991.

61. IFM, Conférence de presse de M. le Président de la République à l'issue du Conseil européen, 10.12.1991.

62. AN, PHB.8-4, PHB. Affaires intérieures et de justice, s.d.

63. AN, PHB.8-4, CDM. Questions institutionnelles (pour le cas où cette question viendrait au Conseil européen), s.d. 
existait un équilibre entre les volets monétaire et économique de l'UEM. La définition de la politique économique relevant in fine du Conseil européen.

Tel que présenté par la présidence néerlandaise, le projet de traité respectait les orientations françaises, avec l'architecture en «Temple» et ses trois piliers. Plusieurs délégations, dont l'Allemagne, emmenées par la Commission, auraient préféré davantage de communautaire, mais la Grande Bretagne s'y opposait résolument. La France avait une position intermédiaire que plusieurs dossiers discutés à Maastricht illustrent parfaitement. ${ }^{64} \mathrm{C}$ 'est d'abord le cas avec la subsidiarité qui permet de mieux répartir les rôles de chacun - régions, États membres, Union européenne - dans l'exercice de cette souveraineté. La France veillait à ce que les États membres aient à dire ce qui relevait, ou non, de l'Union, contrairement à la RFA. C'est ensuite le cas avec l'extension des pouvoirs du Parlement européen. Le projet de traité prévoyait en effet une codécision. C'est-à-dire une procédure de décisions, particulièrement complexe, prolongement de la coopération instaurée par l'Acte unique, qui faisait du Parlement européen - instance démocratique -, un co-législateur au même titre que le Conseil des ministres. Au même titre mais nullement supérieur, puisque Strasbourg obtenait une sorte de droit de veto dans des domaines très précis, mais ne pouvait forcer le Conseil à adopter un texte contre son gré. Les autorités françaises acceptaient cet équilibre.

La RFA et d'autres délégations voulaient étendre cette procédure à un maximum de compétences communautaires, voire octroyer au Parlement un droit de passage en force lors de la conciliation. La Grande-Bretagne ne le voulait pas et Paris, qui ne souhaitait pas apparaître «comme complice de l'obstruction anglaise» attendait simplement que «les Anglais jouer[aient] contre les Allemands».65 Par ailleurs, la délégation française espérait voir la codécision - c'est-à-dire la majorité au Conseil s'appliquer à l'industrie, au social et à la culture. Sur la question de l'avis conforme du Parlement européen, les Français adoptèrent la même prudence. Les députés européens ne pouvaient obtenir un droit permanent d'opposition au Conseil sur de trop nombreux sujets, au risque qu'ils n'abusent de ce droit et entrent dans un chantage budgétaire permanent. L'Allemagne semblait ne pas le craindre et, finalement, la délégation française se contentera d'observer la Grande Bretagne, qui «devrait faire le travail pour nous». ${ }^{66}$ Codécision ou avis conforme, le texte final est à peu près conforme à ce qu'espérait la délégation française. D'une part, l'avis conforme reste limité, d'autre part la procédure de codécision est étendue aux domaines prévus par la France, à l'exception de la culture qui reste du domaine de l'unanimité contrairement aux attentes de Paris. La délégation française, on le verra, échoua aussi à obtenir l'extension de la majorité dans les cas du social et de l'industrie.

64. AN, CDM.14-1, Union politique SN 252/91, 09.12.1991; PHB.8-4, CDM. La structure du traité, 06.12.1991; CDM. Les institutions de la Communauté dans le futur traité d'union politique. Récapitulatif, 25.11.1991.

65. AN, PHB.8-4, CDM, Subsidiarité, s.d.; Pouvoir du Parlement. La co-décision, 06.12.1991.

66. AN, PHB.8-4, CDM. Pouvoir du Parlement. Avis conforme, 06.12.1991. 
Conformément à la dualité du système communautaire qu'elles envisageaient, Paris était aussi à l'origine d'un projet particulier, discuté à Maastricht et intégré au Traité sous forme de déclaration: le Congrès (ou conférence) parlementaire européen. Il s'agissait de réunir ensemble les parlementaires nationaux et européens afin d'adopter de «grandes orientations de l'Union». Le Parlement européen s'y opposait, estimant que cette nouvelle institution empièterait dangereusement sur l'assemblée de Strasbourg, mais la France fut soutenue par plusieurs délégations en ce sens. ${ }^{67}$

Enfin, à l'égard des institutions, les deux derniers problèmes furent réglés - ou plutôt évacués -lors du Conseil européen de Maastricht. Prévoyant de futurs élargissements, plusieurs délégations s'interrogeaient sur le nombre de Commissaires européens nécessaires. ${ }^{68} \mathrm{Ne}$ fallait-il pas revoir les règles de répartition afin de limiter le nombre de commissaires? Les grands États ne devaient-ils pas abandonner leurs seconds représentants dans cette institution? Les petits États y étaient favorables. $\mathrm{La}$ Grande Bretagne y voyait un moyen d'affaiblir la supranationalité de la Commission. Bien qu'initialement isolée sur ce sujet, la France, soutenue par l'Espagne, réussit à faire reporter cette décision à plus tard. La même question se posait, mais de façon plus insistante et plus cruciale, à l'égard du nombre de députés européens. ${ }^{69}$ Désormais unifiée, l'Allemagne réclamait en effet une augmentation du nombre de ses représentants à Strasbourg, notamment pour les Länder de l'Est. Mais ceci revenait à faire de l'Allemagne le pays ayant le plus de députés et brisait donc l'équilibre entre les grands existant sur ce point. Potentiellement, il s'agissait d'une «énorme concession» à la RFA. La France proposa une alternative: une augmentation égale des députés des grands. Mais, après avoir tergiversé, Kohl refusa cette proposition. ${ }^{70} \mathrm{Le}$ traité de Maastricht se contente de renvoyer le problème à la fin de l'année 1992, en même temps que le siège de la BCE.

Enfin, un dernier point, plus ou moins lié à l'architecture du traité, fut discuté en séance. L’Union aurait-elle, ou non, une «vocation fédérale» comme le texte de la présidence néerlandaise le prévoyait? Il s'agissait, pour le Royaume-Uni, d'un véritable «chiffon rouge». ${ }^{71}$ D'ailleurs, à la veille du Conseil européen, la délégation française acceptait déjà son retrait, considérant l'expression «à peu près vide de contenu» dans la mesure où seul le fonctionnement des traités pouvait définir ce qu'était l'Union. Une formule alternative était déjà prévue et permit à Major une victoire à peu de frais.

67. AN, CDM.14-1, Union politique SN 252/91, 09.12.1991; PHB.8-4, CDM. Le congrès, 06.12.1991; PHB.9-1, Casa. Conférence intergouvernementale: Conseil restreint du mercredi 27 novembre, 26.11.1991; TUE.

68. AN, PHB.8-4, CDM. Nombre de commissaires, 06.12.1991.

69. AN, CDM.14-1, Union politique SN 252/91, 09.12.1991; PHB.9-1, EG, Maastricht, 18.11.1991; CDM et PHB. Conseil européen de Maastricht. Demandes allemandes, 21.11.1991; PHB.8-4, CDM. Nombre de membres du Parlement européen. Députés allemands, 06.12.1991.

70. AN, CDM.14-1, Union politique SN 252/91, 09.12.1991; PHB.8-4, CDM. Nombre de membres du Parlement européen. Députés allemands, 06.12.1991.

71. Antici, op.cit.; AN, 14-1, Union politique SN 252/91, 09.12.1991; PHB.8-4, CDM. La vocation fédérale, 06.12.1991. 
Si les questions d'architecture furent discutées, elles n'étaient pourtant pas essentielles pour Paris qui espérait surtout qu'elles n'empêchent pas un accord final. En réalité, en plus des deux sujets majeurs, UEM et PESC, la délégation française avaient deux priorités concernant d'autres politiques communes: le social et l'industrie. $^{72}$ Question moins vitale, elle comptait aussi batailler sur la culture et savait qu'elle devrait prendre position sur la cohésion et la citoyenneté.

Le social est une antienne française depuis 1981. Devant l'opposition de leurs principaux partenaires, Paris et Mitterrand lui-même, cherchèrent à atteindre progressivement leur objectif. En décembre 1989, onze pays adoptaient ainsi une Charte communautaire des droits sociaux fondamentaux des travailleurs fixant les grands principes du droit du travail dans la CEE. Mais Margaret Thatcher avait refusé son accord et le texte était resté en dehors de la juridiction communautaire. Il fallait franchir une nouvelle étape. La France espérait ainsi que le futur traité d'Union comporterait spécifiquement un titre social et que les décisions y seraient prises à la majorité. Il s'agissait de l'une «des principales demandes françaises». Les discussions furent complexes. Plusieurs textes circulèrent et furent discutés en séance plénière. La présidence néerlandaise avait fait une première proposition qui, certes, était une «énorme édulcoration» des ambitions françaises mais avait le mérite de satisfaire l'Espagne. ${ }^{73}$ Ce pays, en effet, craignait que l'adoption de directives sociales ne relève le coût du travail dans son pays, diminuant sa compétitivité au sein de la Communauté. Or, Felipe Gonzales considérait le texte de la présidence comme «un minimum bien équilibré». Paris se savait en mauvaise posture sur ce dossier. La délégation française estimait «indispensable [...] de ramener les Espagnols dans le groupe des 11 pays qui pourr[aient] approuver le texte» et de «vaincre l'intransigeance espagnole». Le projet néerlandais était le seul atteignable à onze. Affaibli, il comportait toutefois un titre social et surtout un large protocole qui faisait référence à la Charte sociale. ${ }^{74}$

Restait le problème britannique. La présidence chercha à présenter un nouveau texte, encore moins ambitieux. Major le refusa malgré l'appel à la modération d'Andreotti. Surtout, d'autres délégations s'y opposèrent, jugeant, comme Jacques Santer, que le premier projet était «un minimum», que la «dimension sociale» était indispensable et que «l'opinion publique ne comprendra[it] pas» son absence. Mitterrand fit de même, déclarant que «la note diffusée [par la présidence dans l']aprèsmidi contredisait le texte [initial]»! L'opposition était totale. Paris savait d'ailleurs que, sur cette question, Major se «battrait jusqu'à la mort» et qu'il s'agissait là de «la seule question qu'il [ne lui était] pas possible de négocier». ${ }^{75}$ Esquissée dans le courant du mois de novembre, précisée à Maastricht même, l'accord sur le volet social devrait donc se faire sans la Grande Bretagne à qui on proposerait une clause d'opting out et qui se trouverait ainsi isolée comme dans le cas de l'UEM. «Hérétique, dan-

72. AN, PHB.9-1, Casa. Conférence intergouvernementales: Conseil restreint du mercredi 27 novembre, 26.11.1991.

73. AN, PHB.8-4, PHB. Politiques sociales, s.d.; CDM.14-1, Union politique SN 252/91, 09.12.1991.

74. Antici, op.cit.; AN, PHB.9-1, Boissieu. Conseil européen: union politique, 22.11.1991; TUE.

75. Antici, op.cit.; AN, PHB.9-1, Casa. Conférence intergouvernementale: Conseil restreint du mercredi 27 novembre, 26.11.1991; PHB.8-4, PHB. Politique sociales, s.d. 
gereuse», créant «un précédent» dans le droit communautaire et donnant éventuellement un «avantage de compétitivité» à la Grande-Bretagne, cette solution semblait la seule possible. La France donna donc son accord, après d'ultimes tractations y compris le dernier jour, à la rédaction d'un protocole ad hoc, excluant la Grande Bretagne et constatant que «que onze États membres [était] désireux de poursuivre dans la voie tracée par la Charte sociale de 1989».76

Éloignée de ses objectifs initiaux, la délégation française obtenait néanmoins satisfaction sur l'inscription d'un titre social. Mitterrand ne pouvait cependant que constater: «nous avons une Europe idéale en vue, mais nous n'y sommes pas». ${ }^{77}$

Second dossier prioritaire, celui dit de l'industrie. «L'un des sujets les plus importants pour la France, qui est pratiquement isolée». ${ }^{78}$ Tous les partenaires de la France s'opposaient en effet à sa tradition interventionniste. Il s'agissait donc pour Paris d'obtenir que la future Union puisse prendre les mêmes décisions que précédemment dans ce domaine - c'est-à-dire d'inscrire un titre « Industrie » dans le traité et, éventuellement, d'étendre les possibilités d'action à la majorité qualifiée. Le projet de la présidence néerlandaise répondait à ces préoccupations, sous réserve de compléments. Mais l'affaire était loin d'être réglée. L'Allemagne, la Grande Bretagne, les Pays-Bas, l'Irlande et le Luxembourg s'y opposaient. C'est d'ailleurs d'Allemagne que vinrent les coups les plus durs. Au final, le titre figure effectivement dans le Traité. L'Union veillerait à son développement via ses outils traditionnels tout en coordonnant l'action des États. Toutefois, de nouvelles mesures spécifiques dans ce domaine ne pouvaient intervenir qu'à l'unanimité. ${ }^{79}$ Bref, l'accord obtenu était ad minima. On notera cependant que le traité octroie de nouvelles compétences à l'Union (santé, environnement, réseaux transeuropéens, protection des consommateurs), soutenues par la France, qui auraient pu ouvrir la voie à de nouvelles interventions auprès des acteurs économiques.

Point jugé moins fondamental que les précédents, la culture n'en était pas moins «une des priorités "sectorielles"». ${ }^{80}$ En 1991, la politique culturelle était encore balbutiante au niveau communautaire, la France étant à l'origine de nombreuses initiatives. Pour Paris, l'objectif était d'inscrire cette dimension en tant que titre à part entière du traité et de permettre des décisions à la majorité. Mais les traditions des autres États dans ce domaine étaient très différentes. Beaucoup craignaient l'action de la Commission dans un domaine où, par ailleurs, les enjeux économiques étaient de plus en plus importants. Les négociateurs français avaient d'ailleurs veillé à isoler la culture des règles de libre-concurrence au nom de la spécificité de cette activité. Rapidement évoqué lors du Conseil européen, le texte adopté était conforme aux

76. AN, PHB.9-1, Boissieu. Conseil européen: union politique, 22.11.1991; PHB.8-4, PHB, Politique sociales, s.d.; CDM.14-1, CDM. Note manuscrite, s.d.; PHB.9-1, CDM, Entretien du Président de la République avec le Chancelier Kohl (Maastricht, mardi 10.12.1991, 16h00), s.d.; TUE.

77. IFM, Conférence de presse ..., op.cit.

78. AN, PHB.8-4, PHB. Industrie, s.d.

79. AN, CDM.14-1, Union politique SN 252/91, 09.12.1991; CDM.14-1, CDM. Note manuscrite, s.d.

80. AN, PHB.8-4, CDM. Culture, 06.12.1991. 
attentes françaises sauf sur un point important: les décisions se prendraient à l'unanimité.

Quant au dossier cohésion, il recouvre en fait une demande espagnole, soutenue par les États membres dits «moins prospères». ${ }^{81}$ Grâce à l'inscription de nouvelles politiques communes et d'un titre ad hoc dans le traité, il s'agissait de modifier les règles de financement et de dépense de la Communauté afin d'accentuer les transferts budgétaires des pays riches vers les moins prospères. Madrid affirmait ne pouvoir signer le futur traité sans avoir gain de cause sur ce sujet, arguant qu'il faudrait de nouvelles aides structurelles pour que certains pays parviennent à remplir les critères sur l'UEM. La Grande Bretagne mais aussi la RFA refusaient ces demandes, jugées trop coûteuses. Cherchant le compromis, le texte de la présidence néerlandaise comportait un titre intitulé «Cohésion économique et sociale» qui permettait d'aider des régions «moins favorisées» - et non des États - et proposait des politiques nouvelles accentuant les transferts. Madrid estimait cependant le texte insuffisant alors que Bonn et Londres trouvaient qu'on allait trop loin. L'affaire fut largement évoquée en séance plénière. Quant à la délégation française, elle espérait trouver une position médiane. Satisfaire l'Espagne - afin de faire front à onze face à la Grande Bretagne sans créer de dépenses qui alourdiraient considérablement sa propre contribution. L'idée était de convaincre Madrid que l'inscription d'un titre suffisait et que la question des transferts serait de nouveau discutée lors de la négociation du Paquet Delors II en 1992. C'est, au final, l'accord qui fut trouvé, le traité de Maastricht actant néanmoins la création de plusieurs politiques jugées redistributives - environnement, réseaux transeuropéens - dont Paris se satisfaisait par ailleurs.

Dernier point, la création d'une "citoyenneté» européenne. ${ }^{82}$ L'idée espagnole était immédiatement soutenue par Paris. La délégation française y tenait. Son contenu restait limité - libre circulation, droit de séjour, droit de vote, protection consulaire commune-mais symboliquement, l'UE ne serait plus un simple marché économique. L'affaire n'était pourtant pas simple. Comme dans le cas du pilier de la Justice et des affaires intérieures (JAI), il touchait à des éléments de la souveraineté nationale. Rapidement abordé en séance plénière du Conseil, le texte final maintint à cet égard le vote à l'unanimité, alors que l'Espagne voulait la majorité, soutenue par la délégation française.

\section{Conclusion - la campagne référendaire}

«En avril 1990, le Chancelier Kohl et moi même, avions proposé à nos partenaires de constituer une union politique. Aujourd'hui, nous avons à Douze, décidé de lui donner vie». ${ }^{83}$ Cette phrase de Mitterrand, par laquelle il entame sa conférence de

81. AN, PHB.8-4, PHB. La cohésion, 06.12.1991.

82. AN, PHB.8-4, CDM. Citoyenneté (articles A à F, pp.15-18), s.d.

83. Conférence de presse ..., op.cit. 
presse le 11 décembre 1991, à 2 heures du matin, résume à elle seule la négociation et les résultats du Conseil européen de Maastricht.

Rien n'aurait été possible sans l'accord franco-allemand. Dans les semaines et les jours qui précédèrent ce Conseil et dans son déroulement, les autorités des deux pays se consultèrent plusieurs fois. Le Président français demanda par exemple à rencontrer le Chancelier pour forcer la négociation vers 16 heures, le 10 décembre, estimant que la présidence néerlandaise était trop complaisante avec la Grande Bretagne. ${ }^{84}$ Ceci fut d'ailleurs vrai à tous les niveaux, jusqu' aux conseillers. Ce travail de couloir, poursuivant les discussions plénières, fut certainement déterminant, même si les archives écrites n'en rendent pas ou peu compte. ${ }^{85}$

$\mathrm{Au}$ terme de cette négociation, on peut dire que les autorités françaises jouèrent un rôle central dans l'élaboration de l'accord final, pour au moins deux raisons. En premier lieu, la délégation française avait fait ses priorités des deux principaux volets du futur traité - l'UEM et la PESC. Son poids politique, allié à celui de l'Allemagne, permit d'aboutir à un texte substantiel, faisant de Maastricht un acte de grande portée, peut-être le «plus important depuis le traité de Rome de $1957 » .{ }^{86}$ En second lieu, parce ce que sa position était souvent pivot entre celles de l'Allemagne et de la Grande-Bretagne, la délégation française put se présenter comme recours dans nombre d'arbitrages.

L'UE était donc née. Il fallait cependant la faire grandir puisque Maastricht restait un compromis. Sur l'UEM, la France avait obtenu ce qu'elle voulait. Sur d'autres sujets, Paris avait dû faire des concessions. Minimes sur la PESC, mais la perspective d'une défense commune existait ainsi qu'un début de vote à la majorité. Minimes sur le social, puisque l'acquis était préservé et que la Charte faisait une entrée timide dans le droit communautaire. Plus importantes sur l'industrie. Sur d'autres thèmes plus secondaires, l'accord lui était favorable. Du reste, et il faut le rappeler, il s'agissait bien à Maastricht de faire enregistrer des avancées à confirmer par la suite. L'Europe à la française restait à inventer ..., ce qui rendit la ratification du traité d'autant plus difficile et alimenta nombre de critiques.

La presse, française et étrangère, ne s'y était pas trompée. Beaucoup de commentateurs saluèrent l'effort, mais tous insistèrent sur les concessions réalisées et les interrogations à venir. Les réactions étrangères, et d'abord allemandes, furent d'ailleurs scrutées à Paris, tout comme l'avancée des différents processus de ratification.

En France, le mercredi 3 juin 1992, au lendemain du référendum danois négatif sur Maastricht, Mitterrand annonça en Conseil des ministres que les Français seraient eux-mêmes appelés à se prononcer directement sur ce sujet. Il le faisait contre l'avis de ses proches ministres, de plusieurs de ses collaborateurs, y compris ses communicants. Ce fut sa dernière bataille politique. Il la gagna de justesse, à la suite d'un

84. AN, PHB.9-1, CDM. Entretien du Président de la République avec le Chancelier Kohl, op.cit.

85. Cf. entretiens de Caroline Margerie et de Pierre Morel; AN, CDM.14-1, CDM. Article D sur la défense, 09.12.1991.

86. AN, PHB.9-1, Lettre de Matignon. 
débat de très grande qualité, qui mobilisa l'attention des Français, notamment lors d'une grande émission télévisée de plusieurs heures, le 3 septembre, où le Chancelier Kohl intervenait personnellement.

Au-delà des considérations politiciennes qu'on ne négligera pas, il nous semble que Mitterrand avait parfaitement mesuré la portée de ce texte, son équilibre, les compromis réalisés et ce qu'il engageait pour son pays. Raison pour laquelle il décida de consulter le peuple français. Le dimanche 20 septembre 1992, le oui l'emporta par $51,04 \%$ des suffrages exprimés. Au même moment, l'Europe se trouvait secouée par une terrible crise monétaire. 


\section{The European Community 1973-1983 approximate}

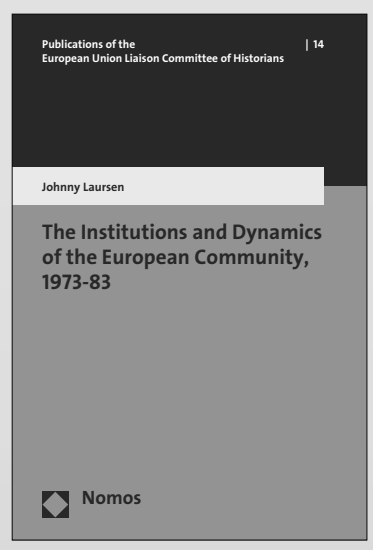

In this volume a group of international historians take a fresh look at this period of European integration. Based on recent archival research and interviews the volume offers a new look on the history of this distinct époque of European integration. Overall, the contributions take up a reinterpretation of the period as a period of crisis and stagnation and offer a discussion of the importance of the developments in the period for the resurgence of the integration process in the subsequent époque of Commission president Jacques Delors.

\section{The Institutions and Dynamics of the European Community, 1973-83}

By Johnny Laursen

2013, approx. 370 pp., pb., approx. € 69.00

ISBN 978-3-8329-6963-9

(Veröffentlichungen der Historiker-

Verbindungsgruppe bei der Kommission $\operatorname{der} E G$, vol. 14)

Approximate publication in August 2013

The book is organised in two main parts: First, four chapters discuss the interpretation of the main dynamics of the integration process with a particular focus on the role of the European institutions. The second part provides new interpretations of the emergence of monetary co-operation and of the emergence of new policy areas and international challenges in European co-operation. 